\section{Interaction of atomic systems with X-ray free-electron lasers}

\section{A. Kornberg, ${ }^{a}$ A. L. Godunov, ${ }^{b}$ S. Itza-Ortiz, ${ }^{b}$ D. L. Ederer, ${ }^{b}$ J. H. McGuire ${ }^{b *}$ and L. Young ${ }^{c}$}

${ }^{a}$ Max-Planck-Institut für Physik Komplexer Systeme, Nöthnitzer Strasse 38, D-01187 Dresden, Germany, ${ }^{b}$ Department of Physics, Tulane University, New Orleans, LA 70118-5698, USA, and ${ }^{C}$ Argonne National Laboratory, Argonne, IL 60439, USA. E-mail: mcguire@mailhost.tcs.tulane.edu

The planned construction of an X-ray free-electron laser (XFEL) will provide new opportunities for research in various areas of physics, chemistry and biology. The proposed design of the XFELs at DESY (Deutsches Elektronen-Synchrotron) and SLAC (Stanford Linear Accelerator Center) is built on the concept of a fourth-generation synchrotron source and will provide an intense pulse $\left(I_{0} \simeq\right.$ $10^{16} \mathrm{~W} \mathrm{~cm}^{-2}, \tau_{\gamma} \simeq 100 \mathrm{fs}$ ) for photon wavelengths down to $1 \AA$. Some guidelines for applications of these sources pertaining to atomic physics are presented here. Issues such as the onset of strong photon-field effects, multiple ionization and hollow-atom formation are analyzed. Attention is especially given to studying the interaction with rare-gas atoms, for which some numerical estimates are provided.

Keywords: free-electron lasers; photoionization; strong-field effects; hollow atoms; multiple ionization.

\section{Introduction}

Since their discovery in 1945 (Blewett, 1946), synchrotron radiation sources have contributed to our understanding of many phenomena in the areas of physics, chemistry and biology. The technical development of synchrotron sources has evolved through three generations, each providing higher brightness than their predecessors (Winick, 1998). Third-generation sources have provided an opportunity for experiments at high photon energies, presently extending up to $100 \mathrm{keV}$ (Tschentscher \& Suortti, 1998).

Presently, we are facing a breakthrough in the technology of synchrotron sources, which is leading to the development of fourthgeneration machines in the X-ray regime, also known as the X-ray free-electron laser (XFEL). While these sources are still built according to the concept of a synchrotron source, the beam properties are mainly those of a laser source, with wavelengths extending well into the X-ray region. Two well known projects at DESY (DESY, 2001; Brinkmann et al., 1997) and SLAC (SLAC, 2001; Tatchyn et al., 1996) plan to provide photon intensities of about $10^{16} \mathrm{~W} \mathrm{~cm}^{-2}$ with photon wavelengths down to $1 \AA$. The technical status of shortwavelength free-electron lasers up to 1996 was summarized by Colson (1997), and the shortest wavelength attainable at that time was reported to be $250 \mathrm{~nm}$.

In this work, we are concerned with the impact of XFEL sources on studies of basic atomic physics, primarily in rare gases. This is a narrow segment of the full range of applicability of XFELs. However, this segment is relevant because the XFEL wavelength is small enough to reach into the inner atomic core of the various materials probed. Moreover, the first atomic physics experiments are likely to be performed using noble gases. A general overview of the wide applicability of XFELs was undertaken by Brinkmann et al. (1997), covering material science, biology and molecular physics, among others. Other studies on specific areas have also begun to appear in the literature (Doniach, 2000). At the atomic level, XFELs will interact with all forms of matter. Recently, there have been two papers published in atomic physics that are concerned with ionization by a strong source within the XUV region (Brewczyk \& Rzazewski, 1999; Gajda et al., 2000). To our knowledge, no work has been published so far that considers the interaction of atomic systems with photon sources having the characteristics of the XFEL. In this work, we will consider the specific characteristics of the XFEL sources planned at DESY and SLAC. However, our discussion is quite general and many considerations are equally applicable to other sources planned to operate in the XUV and soft-X-ray region (Colson, 1997). The principal characteristics of the sources at DESY and SLAC are summarized in Table 1. For our applications on atomic physics, relevant parameters are wavelength, pulse duration, spot size and number of photons per pulse. Despite differences in design, construction and performance, as is indicated for the different cases in Table 1, we will assume the following values for our analysis: wavelength $1 \AA$, pulse duration $100 \mathrm{fs}$, spot size $20 \times 20 \mu \mathrm{m}$ and number of coherent photons per pulse $5 \times 10^{12}$. In some cases, we will discuss the effects of varying some of these parameters.

The purpose of our work is therefore twofold. On one side, we will give some general considerations for atomic systems, which we feel to be of wide interest for future applications. The area of hollow atoms is of special interest, as is consideration of multielectron processes in the quite unusual characteristics of the XFEL. On the other hand, we wish to provide some initial estimates for ionization of rare gases with XFEL sources and to point out issues of interest in this field. General conclusions concerning the issues studied will be given in the last section.

\section{Topics of interest for atomic systems}

In this section, we will give an outline of some basic issues to be considered concerning the interaction of radiation from an XFEL with an atomic environment. The discussion will be mostly qualitative at this stage, since we are trying to identify issues that we feel are most interesting. In the next section, we will use some concrete examples on the rare gases to set down quantitative criteria for future work. We divide the discussion into subsections of special interest.

\subsection{Onset of strong photon-field effects}

Studies on the interaction of hard-X-ray photons with atomic targets have been entirely in the weak-field regime. In an XFEL, the strong-field regime may be approached. Here we make some estimates to characterize the strong-field regime and relate them to the design parameters of the XFEL: wavelength $\lambda$, pulse duration $\tau_{\gamma}$, spot size $A$ and number of coherent photons per pulse $N_{\gamma}$.

To characterize the strong-photon-field regime, we require that the electric field of the photons $\left(E_{\gamma}\right)$ be comparable with the electric field binding an electron of principal quantum number $n$ to a target nucleus of charge $Z$, namely

$$
E_{\gamma} \simeq E_{\mathrm{cr}}(Z, n) \quad \text { (strong field boundary). }
$$

For a hydrogenic electron, the binding energy $U_{\text {binding }}(Z, n)$ scales as $Z^{2} / n^{2}$, and the orbital radius $r_{\text {orbit }}(Z, n)$ scales as $n^{2} / Z$. Thus, the critical field strength for $Z$ targets is given by

$$
E_{\text {cr }}(Z, n)=U_{\text {binding }} / r_{\text {orbit }}=\left(Z^{3} / n^{4}\right) E_{\text {cr }}(Z=1, n=1),
$$

where the critical field for hydrogen is $E_{\mathrm{cr}}(Z=1, n=1)=$ $2 \times 13.6 \mathrm{~V} / 0.529 \AA=5.14 \times 10^{9} \mathrm{~V} \mathrm{~cm}^{-1}$. 
Table 1

Parameters for three cases of the DESY project (Brinkmann et al., 1997) and for three cases of the SLAC project (Tatchyn et al., 1996).

\begin{tabular}{|c|c|c|c|c|c|c|}
\hline XFEL & DESY-1 & DESY-2 & DESY-3 & SLAC-1 & SLAC-2 & SLAC-3 \\
\hline Wavelength $\lambda(\AA)$ & 1 & 2.4 & 1 & 40 & 4.5 & 1.5 \\
\hline Pulse length $\tau_{\gamma}$ (fs) & 80 & 80 & 80 & 300 & 150 & 250 \\
\hline Coherent photons per pulse $\left(\times 10^{13}\right)$ & 0.3 & 0.8 & 0.8 & 6.6 & 3.3 & 0.5 \\
\hline Transverse size $(\mu \mathrm{m})$ & 25 & 28 & 17 & 80 & 30 & 20 \\
\hline Peak current (kA) & 5.0 & 5.0 & 5.0 & 2.5 & 5.0 & 5.0 \\
\hline Electron beam energy $(\mathrm{GeV})$ & 25 & 25 & 50 & 7 & 15 & 15 \\
\hline Undulator period $\lambda_{0}(\mathrm{~cm})$ & 5.0 & 7.0 & 7.0 & 8.3 & 4.0 & 3.0 \\
\hline Repetition rate $(\mathrm{Hz})$ & 50 & 50 & 50 & 120 & 120 & 120 \\
\hline Peak power $(\mathrm{GW})$ & 65 & 85 & 200 & 10 & 100 & 50 \\
\hline Average power $(\mathrm{kW})$ & 0.8 & 1.0 & 2.4 & 0.4 & 1.4 & 1.6 \\
\hline Bandwidth (FWHM) (\%) & 0.2 & 0.2 & 0.2 & 0.1 & 0.1 & 0.1 \\
\hline Peak brightness $\dagger\left(\times 10^{31}\right)$ & 660 & 350 & 2000 & 5 & 500 & 500 \\
\hline Average brightness $\dagger\left(\times 10^{23}\right)$ & 800 & 420 & 2400 & 0.02 & 1 & 1 \\
\hline Divergence angle $(\mu \mathrm{rad})$ & 0.75 & 1.4 & 0.8 & 25 & 10 & 5 \\
\hline Normalized emittance (mm mrad) & 3.0 & 3.0 & 3.0 & 3.5 & 1.0 & 1.0 \\
\hline Saturation length (m) & 95 & 87 & 95 & 60 & 40 & 55 \\
\hline Peak magnetic field $(\mathrm{T})$ & 0.89 & 0.85 & 0.78 & 0.76 & 1.6 & 1.3 \\
\hline
\end{tabular}

$\dagger$ In units of photons $\mathrm{s}^{-1} \mathrm{~mm}^{-2} \mathrm{mrad}^{-2}(0.1 \% \text { bandwidth })^{-1}$.

Now we turn our attention to the field strength of the photons in the XFEL. The power density for the XFEL is given by

$$
I_{\gamma}=N_{\gamma}(h c / \lambda)\left(1 / A \tau_{\gamma}\right)
$$

For the nominal specifications $\tau_{\gamma}=100 \mathrm{fs}, N_{\gamma}=5 \times 10^{12}$ photons per pulse, $A=4 \times 10^{-6} \mathrm{~cm}^{2}$ at a wavelength $\lambda=1 \AA$, the power density is $2.5 \times 10^{16} \mathrm{~W} \mathrm{~cm}^{-2}$. This can be easily converted to an electric field strength, assuming linearly polarized fields, using the relationship $I_{\gamma}\left(\mathrm{W} \mathrm{cm}^{-2}\right)=\epsilon_{0} c\left[E_{\gamma}\left(\mathrm{V} \mathrm{cm}^{-1}\right)\right]^{2}$.

This yields a field strength of $4.3 \times 10^{9} \mathrm{~V} \mathrm{~cm}^{-2}$, which is comparable to the critical field for hydrogen. Since the critical field for higher- $Z$ targets scales as $Z^{3} / n^{4}$, it will not be possible to reach the strong-field regime for inner shells $(n=1)$ of intermediate- $Z$ targets. However, with effective focusing, for example, a demagnification factor of 4000, one may approach the strong-field regime for $\mathrm{Ne}$, $Z=10, n=2$.

We note that it is possible to detect strong-field effects when the photon field is somewhat weaker than that estimated above. Of course, strong-field effects are usually relatively small unless the transition is forbidden (or at least inhibited) under weak conditions. We also note that in the weak-field regime the incident photon intensity and yield vary as $E_{\gamma}^{2}$, but this is not the case in the strongfield regime. In the strong-field regime a variety of new physics comes into play, including non-linear effects, non-dipole effects, entanglement of one or more electrons with the photon field, and multipleelectron transitions, especially multiple ionization.

\subsection{Multiple ionization}

A question of fundamental importance in plasma formation is how multiple ionization occurs in strong fields. Experiments have been performed previously for long wavelengths [cf. a review for twoelectron atoms by Lambropoulos et al. (1998); also Parker et al. (2001)], and the mechanisms for production of multiply charged ions are considered to be a sequential or a direct transition (Crance, 1987). In most cases, the sequential transition dominates, and sequential models based on an independent-particle approximation (IPA) were developed by Geltman (1985).

We consider first the case of the sequential transition, for which the independent-particle assumption can be applied. In the IPA, the electrons are stripped independently from the atom by the strong field. Since the collision time is slow compared with the electronic orbit time, there is time for the electrons to readjust. Therefore, each stage of ionization occurs with a different binding energy and screening parameter. For a system with $N$ independent electrons, the probability is a factor of single-electron terms, e.g. a multinomial distribution,

$$
P=P_{1} P_{2} P_{3} \ldots\left(1-P_{N-1}\right)\left(1-P_{N}\right) .
$$

Here $P_{j}$ is the probability for removing a single electron by the strong field and $\left(1-P_{j}\right)$ is the probability that the electron is not ionized. In this model, the difficulty of managing the $N$-electron problem is reduced to a simpler one-electron problem solved $N$ times with $N$ different binding energies. If the single-electron probabilities are nearly equal, $P_{j} \simeq P$, and a simple binomial expression of the form $P^{n}(1-P)^{N-n}$ results for the ionization of $n$ of the $N$ electrons. One might expect that the transition from single to multiple ionization would occur rapidly owing to factors of $P^{n}(1-P)^{N-n}$. As $P \rightarrow 1$, the $(1-P)^{N-n}$ terms go quickly to zero, and it is unlikely that many electrons are left in the atom. However, this rapid transition from the weakly ionized to the strongly ionized state occurs more slowly, since the transition is sequential, and the binding energies adjust so saturation for each stage of ionization tends to occur at different field strengths depending on the ionization potential at that stage of ionization. Saturation tends to occur when the photon-field strength is greater than the binding to the atom by its nucleus (Walker et al., 1994; Becker \& Faisal, 1999).

Application of the independent-particle assumptions, in the form outlined above, could be straightforward for the conditions of the XFEL, as seen previously in other regimes (Geltman \& Zakrzewski, 1988). One significant issue to take into account is the influence of shake-off contributions, which are quite important in the high-energy region considered here. The shake-off contribution is here the name for the 'direct' transition, which is characteristically different from the 'sequential' picture outlined above. While direct transitions for formation of multiply charged ions are difficult to calculate for multiphoton processes (Crance, 1987; Lambropoulos et al., 1998), the shake-off contribution for high photon energies is a well known and widely studied topic (Åberg, 1994; McGuire, 1997). In strong fields in the XUV and X-ray regime, the competition between sequential (independent-electron) transitions and direct (shake-off) transitions comes into play (Becker \& Faisal, 1999). In $\$ 3$ we will analyze the situation for the conditions of the XFEL and for rare-gas atoms. However, and even more importantly, the mechanisms of multiple 
ionization are very different for IR lasers and X-rays. X-rays create inner-shell vacancies, which then automatically create multiply charged ions by Auger decay, radiative transitions and shake processes. For example, the dominant charge state for irradiating $\mathrm{Ar}$ with a $10 \mathrm{keV}$ photon would be $\mathrm{Ar}^{4+}$. The initial photoionization of the $K$-shell electron would be followed by rapid Auger decay, first the $K-L L$ to produce $2+$ and then the $2(L-M M)$ to produce $4+$ (Kochur et al., 1995).

\subsection{Hollow atoms}

In the hard X-ray range, the probability for ionization of atomic inner shells can be greater than the probability for ionization of outer shells. If this occurs, then the atom may be hollow if the outer shells still have some electrons. The term 'hollow atom' has been used since 1990 (Briand et al., 1990) to denote this situation, but study of these interesting cases in atomic physics has been scarce within the area of photon-atom interactions. Hollow atoms have been mainly produced in surface-ion collisions (Winter \& Aumayr, 1999), and it is in that context that they have been largely studied. A special case within photon-atom interactions that deserves to be mentioned is the case of lithium, where it is feasible to excite the inner shell with today's technology. An Li hollow atom has been studied with synchrotron radiation (Kiernan et al., 1994; Azuma et al., 1995; Journel et al., 1996) and also theoretically in a multiphoton context (Madsen et al., 2000). Recently, there have been studies on the production of hollow $K$-shells in intermediate $Z$ atoms using single-photon excitation in the X-ray range. An initial study using solid-state detectors and coincidence techniques (Kanter et al., 1999) was followed by higherresolution studies using X-ray spectrometers (Diamant et al., $2000 a, b)$. Availability of short-wavelength sources such as the XFEL will provide new opportunities for research in this growing field of atomic physics.

The mechanisms of formation and decay of hollow atoms by excitation of an XFEL source will be a prime area of study. The photon pulse length $\left(\tau_{\gamma}\right)$ characterizes in some way the formation of a hollow atom. A hollow atom decays with times characteristic of radiative decay $\left(\tau_{r}\right)$ and Auger decay rates $\left(\tau_{a}\right)$. For the XFEL, we have $\tau_{\gamma} \simeq 100 \mathrm{fs}$. The characteristic times of decay would depend of course on the atom and the state of ionization. However, in general, Auger decay dominates over radiative decay for $1 s$ vacancies in atoms with low $Z$ values (Bambynek et al., 1972). For the case of $\mathrm{Ne}$, $\tau_{r} \simeq 160 \mathrm{fs}$ and $\tau_{a} \simeq 3 \mathrm{fs}$, which is much smaller than $\tau_{\gamma}$. Thus, the typical decay times of hollow atoms are smaller than the typical time of a pulse duration for the conditions of the XFEL. If the excitation and decay times are comparable, then the two processes may not be treated separately, but rather must be treated as a single complex process.

Hollow atoms have been of interest in recent years in connection with the generation of short-wavelength radiation. Two experiments demonstrating the photoionization of inner-shell electrons followed by Auger decay into excited states were reported for $\mathrm{Xe}$ and $\mathrm{Kr}$ (Kapteyn et al., 1986; Kapteyn \& Falcone, 1988), resulting in the production of short-wavelength radiation of about $100 \mathrm{~nm}$. More recently, experiments showing these characteristics in $\mathrm{Kr}$ and $\mathrm{Xe}$ clusters have been reported by McPherson, Luk et al. (1994) and McPherson, Thompson et al. (1994), resulting in the emission of X-ray pulses in the 2-3 $\AA$ range. Further, the possibility of lasing using hollow atoms has been studied for $\mathrm{Na}$ and $\mathrm{C}$ by Moribayashi et al. (1998). Besides the use of hollow atoms as a lasing source, hollow atoms themselves are interesting for their quite unusual properties in comparison with filled atoms. Hollow atoms are Rydberg-like; they carry a lot of energy around, which is released when they decay, e.g. in a subsequent collision where they might deposit their energy selectively.

\subsection{Fast-reaction dynamics}

Within a pulse of an XFEL, a photon is relatively tightly bunched with a coherence length of $\tau_{\gamma}$ that can be as small as $50 \mathrm{fs}$. Ideally this means that if a process takes much longer than $\tau_{\gamma}$ it will lose coherence. Thus, steps longer than $\tau_{\gamma}$ may be regarded as classical (incoherent) events and may be separated. Therefore reaction dynamics may be studied on time scales down to $\tau_{\gamma}$. This may be useful in some chemical and possibly biological reactions. When considering the interaction with $\mathrm{Ar}$, for example, this probably means that radiative decay of an outer electron may be considered separately from the formation of the highly excited states. However, for some other systems, deexcitation of the final state may occur on a time scale comparable with the excitation time, $\tau_{\gamma}$.

A remarkably broad range of electromagnetic pulse widths, $\tau_{\gamma}$, are available to probe fast-reaction dynamics. Time widths of pulses coming from current optical lasers can be as small as a few femtoseconds. Below this limit, the physics changes for visible light since the carrier-wave frequency for visible light is in this range. Waves of shorter duration than this are electromagnetic pulses with no well defined carrier frequency, i.e. definitely not sinusoidal in their nature. However, even $1 \mathrm{fs}$ is 400 times longer than the orbit time for an electron in the ground state of hydrogen, which is approximately the orbit time for valence electrons in all neutral atoms. By this standard, synthetic photon pulses are relatively slow. Electromagnetic pulses 1000 times faster than the Bohr orbit time have been achieved in fast ion-atom collisions using relativistic uranium ions (Moshammer et al., 1997). If the ion is highly charged, the ion fields can be stronger than those in the target atom. Thus, in principle, by using both XFELs and highly charged ions, one may probe atoms with electromagnetic pulses that range from very long pulses (longer than $100 \mathrm{ps}$ ) to pulses shorter than $10^{-19} \mathrm{~s}$.

\section{Estimates for rare-gas atoms}

The interaction of atomic systems with strong laser sources is a relatively well understood topic. The issues range from multiphoton absorption and above-threshold ionization to harmonic generation and stabilization (Gavrila, 1992). These studies have been undertaken for photon wavelengths of the available lasers, i.e. in the IR, UV and, more recently, XUV. However, these issues have not been studied for X-ray energies, since lasers for these wavelengths have not been previously available. Here we consider the issue of photonatom interactions with a strong $\mathrm{X}$-ray source.

In this section, we specifically consider the interaction of radiation from an XFEL with rare-gas atoms. According to recent specifications (Brinkmann et al., 1997; Tatchyn et al., 1996), an XFEL will provide about $5 \times 10^{12}$ photons in $100 \mathrm{fs}$ at a wavelength of $1 \AA$ in a beam of about $20 \times 20 \mu \mathrm{m}$ (see Table 1). Accordingly, the flux is about $F \simeq 10^{31} \mathrm{~cm}^{-2} \mathrm{~s}^{-1}$, and the intensity is $I \simeq 10^{16} \mathrm{~W} \mathrm{~cm}{ }^{-2}$. For photon wavelengths of $1 \AA$ ( $12.398 \mathrm{keV})$, the main contribution to the ionization yield should come from the inner shells, especially the $K$-shell. This is true for $\mathrm{He}, \mathrm{Ne}$ and $\mathrm{Ar}$, although the $L$-shell will be mostly depleted for $\mathrm{Kr}$ and Xe. Energy conservation allows that one photon be enough for ionization, and we may expect that few-photon processes will also be observed under the conditions of the XFEL. We do not expect that there will be much contribution from electron correlation, except in some, mostly weak, multiple-electron transitions (McGuire et al., 2000). For strong fields, the photon fields are 
stronger than the electron-electron fields, and the independentparticle approximation should usually hold (McGuire, 1997).

In strong-field interactions, two different regimes are considered for the ionization process: (a) photoabsorption in a moderate intensity field and $(b)$ tunneling when the field is strong enough for bending the Coulomb barrier. These two regimes are separated by considering the Keldysh parameter (Keldysh, 1965), which is the ratio of the incident field frequency to the tunneling rate,

$$
\gamma=\left(E_{I} / 2 U_{\mathrm{p}}\right)^{1 / 2}
$$

where $E_{I}$ is the ionization potential, and $U_{\mathrm{p}}$ is the ponderomotive potential, $U_{\mathrm{p}}=2 \pi \alpha I / \omega^{2}$, where $\alpha$ is the fine-structure constant and $I$ is the intensity relative to the atomic unit ${ }^{1}$ defined by $6.436 \times 10^{15} \mathrm{~W} \mathrm{~cm}^{-2}$. This subdivision of ionization corresponds physically to multiphoton ionization when $\gamma \gg 1$ and to tunneling when $\gamma \ll 1$. In the case of Ar, for which $E_{I}=118$ a.u., and using $\omega=440$ a.u., the Keldysh parameter $\gamma \simeq 18000$ indicates that we are well into the multiphoton regime.

We estimate the ion yield for the rare-gas atoms by using simple one-photon cross sections (Berger \& Hubbell, 1987). For a given cross section $\sigma_{(Z)}$, writing the pulse shape as $I(t)=I_{0} f(t)$ where $I_{0}$ is the peak intensity, the ionization probability is given by

$$
P\left(I_{0}\right)=1-\exp \left[-\sigma_{(Z)}\left(I_{0} / \hbar \omega\right) \tau_{\text {eff }}\right],
$$

where the effective pulse duration is $\tau_{\text {eff }}=\int_{-\infty}^{+\infty} f(t) \mathrm{d} t$, which for a Gaussian pulse shape $\left\{f(t)=\exp \left[-(t / \tau)^{2}\right]\right\}$ equals $\pi^{1 / 2} \tau$. The parameter $\tau$ can be taken to be about $\tau_{\gamma} / 1.66$, where $\tau_{\gamma}$ is the experimental pulse width, in our case $100 \mathrm{fs}$. We should note that the various definitions of the $\tau$ parameters give similar results here; they differ more for a high- $N$ photon process. The values for cross sections for the rare gases are given by (Berger \& Hubbell, 1987) $\sigma_{(2)}=1.5$ barns for $\mathrm{He}$, taking into account both photoabsorption and Compton scattering; $\sigma_{(10)}=2.0 \times 10^{2}$ barns for $\mathrm{Ne}$, with only a small contribution from Compton ionization; $\sigma_{(18)}=2.2 \times 10^{3}$ barns for $\mathrm{Ar}$ (the cross section for Compton scattering here is two orders of magnitude smaller than for photoabsorption); $\sigma_{(36)}=3.8 \times 10^{3}$ barns for $\mathrm{Kr}$, where the $L$-shell is photoionized; and $\sigma_{(54)}=2.0 \times 10^{4}$ barns for Xe with ionization of the $L$-shell.

In Fig. 1, we plot the ionization yield for the rare gases as a function of peak intensity. The first thing to note is that saturation sets in at much higher intensities than expected for the unfocused XFEL (about $10^{16} \mathrm{~W} \mathrm{~cm}^{-2}$ ). In this case, we are favorably situated to study ionization phenomena in the rare gases, with considerable improvement in the acquisition of data owing to the higher average flux of the XFEL. Generally, in cases where the interaction of rare-gas atoms with pulsed optical lasers is studied, for intensities as high as $10^{15} \mathrm{~W} \mathrm{~cm}^{-2}$, saturation has already set in (Perry et al., 1988). In the case of an XFEL experiment, we are in a region where secondary (or post-collisional) effects could be amply studied. PCI is not very important in the weak-field limit (Åberg, 1994). Finally, we should point out that, except for He, inner-shell ionization leads to a cascade that will result in further ionization of the atom (Kochur et al., 1995). In Fig. 1 the estimates are given for the first step of the cascade.

The ionization yields plotted in Fig. 1 come from single-electron detachment by the absorption of a single photon. The first thing to study is the feasibility of observing a two-photon process within the proposed conditions. In this case, a two-photon process will be related

\footnotetext{
${ }^{1}$ We note that the intensity of a photon field at $E=E_{1}$ is $I_{1}=3.5 \times 10^{16} \mathrm{~W} \mathrm{~cm}^{-2}$, while the atomic unit of intensity is $I_{\gamma}=6.4 \times 10^{15} \mathrm{~W} \mathrm{~cm}^{-2}$, corresponding to $I_{1}=I_{\gamma} / 8 \pi \alpha$, with $\alpha$ the finestructure constant.
}

to an inner-shell absorption, contrary to the case generally studied where electrons are stripped from valence or outer shells. Theoretically, a calculation in this case would be more demanding. The transition by two-photon absorption in the lowest order of perturbation theory implies the calculation of an amplitude $t_{f i}^{(2)}$ that involves a summation over a complete set of intermediate states $\left|\Psi_{a}\right\rangle$. For the case of high- $Z$ targets, the intermediate states in the sum should be described by methods beyond a frozen-core approximation. This would be complex for the problem at hand. To our knowledge, the only available calculations of this type have been performed recently for the $1 s$ shell of noble gases and highly-charged ions (Novikov \& Hopersky, 2000, 2002a,b), but for photon energies less than $1 \mathrm{keV}$. For the photon energies addressed here $(\omega \simeq 12 \mathrm{keV})$, we will make an estimate based on a hydrogenic approximation. We consider a two-photon process in the $K$-shell of $\mathrm{Kr}$ at $1 \AA$. We can obtain an estimate of the cross section by using a hydrogenic scaling equation (Zernik, 1964),

$$
\hat{\sigma}^{(2)}(\omega, Z)=\left(1 / Z^{8}\right) \hat{\sigma}^{(2)}\left(\omega / Z^{2}, 1\right),
$$

where $\hat{\sigma}^{(2)}(\omega, Z)$ denotes the generalized cross section for a twophoton process for a hydrogenic atom of charge $Z$ at the photon frequency $\omega$. Since for hydrogen we have $\hat{\sigma}^{(2)}(9.3 \mathrm{eV}, Z=1) \simeq$ $1.5 \times 10^{-50} \mathrm{~cm}^{4} \mathrm{~s}$ (Karule, 1990), an estimated value for the case of Kr would be $\hat{\sigma}^{(2)}(12 \mathrm{keV}, Z=36) \simeq 5 \times 10^{-63} \mathrm{~cm}^{4} \mathrm{~s}$. The probability obtained for two-photon absorption for the $K$-shell of $\mathrm{Kr}$ is therefore quite small for the XFEL parameters, where the intensity is $\sim 10^{31}$ photons $\mathrm{cm}^{-2} \mathrm{~s}^{-1}$. However, two-photon ionization/excitation resonances occur in atomic systems (Novikov \& Hopersky, 2000), and the tunability of the XFEL will enable one to access these resonances. Tuning to one of these resonances can enhance the two-photon absorption rate such that it exceeds the single-photon absorption rate. On the other hand, low- $Z$ atoms appear to be the more promising candidates for the observation of few-photon processes owing to the factor $Z^{-8}$ in (7). The properties of the XFEL radiation can also increase the probability of observing few-photon processes; the non-uniformity of the radiation within a bunch and effects from capillary focusing (Brinkmann et al., 1997) may increase the rate for such processes.

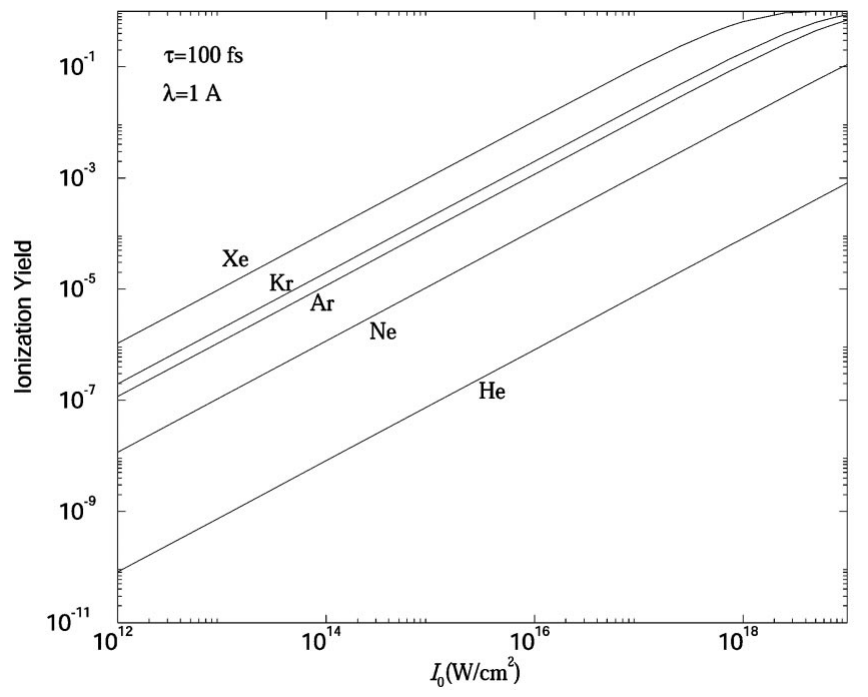

Figure 1

Ionization yield as a function of peak intensity for the rare gases. The photon wavelength is $1 \AA$, and the pulse duration is $100 \mathrm{fs}$. 
A topic of fundamental importance to be studied within the XFEL conditions is multiple ionization. For a high-energy photon in a weak field, multiple ionization occurs due to shake-off (Åberg, 1970). In a strong field, we should inquire about the importance of sequential transitions, which in some cases may eventually dominate. To set the issue in quantitative terms, we will consider ionization with two steps and apply it to He and Xe. The yield of neutrally $\left(P_{0}\right)$, singly $\left(P_{1}\right)$ and doubly $\left(P_{2}\right)$ charged ions is calculated through a set of coupled differential equations (Crance \& Aymar, 1985):

$$
\begin{gathered}
\mathrm{d} P_{0} / \mathrm{d} t=-\left(\gamma_{01}+\gamma_{02}\right) P_{0}, \\
\mathrm{~d} P_{1} / \mathrm{d} t=-\gamma_{12} P_{1}+\gamma_{01} P_{0}, \\
\mathrm{~d} P_{2} / \mathrm{d} t=\gamma_{02} P_{0}+\gamma_{12} P_{1},
\end{gathered}
$$

where $\gamma_{01}$ is the probability of single ionization of the neutral atom, $\gamma_{12}$ is the probability of single ionization of the singly ionized species, and $\gamma_{02}$ is the probability of double ionization of the neutral atom by shake-off. $P_{i}$ are related by the number-conservation relation $P_{0}(t)+P_{1}(t)+P_{2}(t)=1$. The values for $\gamma$ can be obtained from known cross sections, and the shake-off contributions are $1.7 \%$ for He (Åberg, 1970) and 18\% for the $L$-shell of Xe (Carlson \& Nestor, 1973). When the intensity is given in $\mathrm{W} \mathrm{cm}^{-2}$, and the photon energy is $12.48 \mathrm{keV}$, the values are given by

(a) for $\mathrm{He}, \quad \gamma_{01}=5.2 \times 10^{-26} I_{0}, \quad \gamma_{12}=1.9 \times 10^{-26} I_{0}, \quad \gamma_{02}=$ $9.0 \times 10^{-28} I_{0}$ in $\mathrm{cm}^{2} \mathrm{~W}^{-1} \mathrm{fs}^{-1}$;

(b) for $\mathrm{Xe}, \quad \gamma_{01}=1.1 \times 10^{-20} I_{0}, \quad \gamma_{12}=9.0 \times 10^{-21} I_{0}, \quad \gamma_{02}=$ $1.9 \times 10^{-21} I_{0}$ in $\mathrm{cm}^{2} \mathrm{~W}^{-1} \mathrm{fs}^{-1}$.

We note that in the case of He we have only included the contribution by photoabsorption, in order to study in a clear way this contribution in two different atoms. If the Compton contribution is included as well, we do not expect many changes in the features observed in our results. Also, for the case of Xe, the contribution from the Auger decay has not been included.

Putting $P_{0}(t)=1-P_{1}(t)-P_{2}(t)$ into (9) and (10) gives

$$
\begin{aligned}
& \mathrm{d} P_{1} / \mathrm{d} t=-\left(\gamma_{01}+\gamma_{12}\right) P_{1}-\gamma_{01} P_{2}+\gamma_{01}, \\
& \mathrm{~d} P_{2} / \mathrm{d} t=-\left(\gamma_{02}-\gamma_{12}\right) P_{1}-\gamma_{02} P_{2}+\gamma_{02} .
\end{aligned}
$$

We solved (11) and (12) with pulse length $100 \mathrm{fs,} \mathrm{and} \mathrm{we} \mathrm{show} \mathrm{our}$ results in Fig. 2. For the case of $\mathrm{He}$, we note that the shake-off term for the production of $\mathrm{He}^{2+}$ is dominant for the conditions of the XFEL, while the contribution from a sequential transition (marked by the dashed line) is strongly reduced. In the case of Xe, however, the contribution from sequential transitions is more important, but the shake-off contribution is still larger in magnitude for the intensity of the XFEL (about $10^{16} \mathrm{~W} \mathrm{~cm}^{-2}$ ). Note that in the case of $\mathrm{Xe}$ at about $10^{18} \mathrm{~W} \mathrm{~cm}^{-2}$ the shake-off and sequential transitions are of the same magnitude, and in this case interference between both paths would appear. To calculate the shake-off contribution accurately when the strong-field effect is of comparable magnitude, we expect that theories capable of handling both effects on the same footing (shake processes and strong-field effects) would be required. Concerning the inner-shell ionization of $\mathrm{Xe}$, when an initial vacancy is created in the $L$-shell, a cascade will lead to further ionization of the atom, where $\mathrm{Xe}^{8+}$ is the most probable charge state (Mukoyama, 1986; Tawara et al., 1992). As seen in Fig. 2(b), for the first step of this cascade, sequential transitions appear not to be so important for low intensities. At higher intensities, however, strong-field effects come into play, and weak-field calculations of the cascade are no longer applicable.
It should be further stressed that the results of Fig. 2 are only specific cases. Substantial differences would also probably be observed if the conditions of intensity and pulse duration were varied or if other atoms were used. One reason that the shake-off term is important in the results of Fig. 2 is the short duration of the pulse, since in the solutions of (11) and (12) we observe that $P_{2}(t) \simeq \gamma_{02} t$ for $t \rightarrow 0$. Increasing the pulse duration would in fact introduce substantial modifications, and it could change the relative contributions of the shake-off and the sequential transition, even for the same parameters of the laser and atom.

\section{Conclusions}

In this work we have addressed the issue of the interaction of atomic systems with X-ray radiation, as is expected to be obtained from the future fourth-generation synchrotron sources at DESY and SLAC.

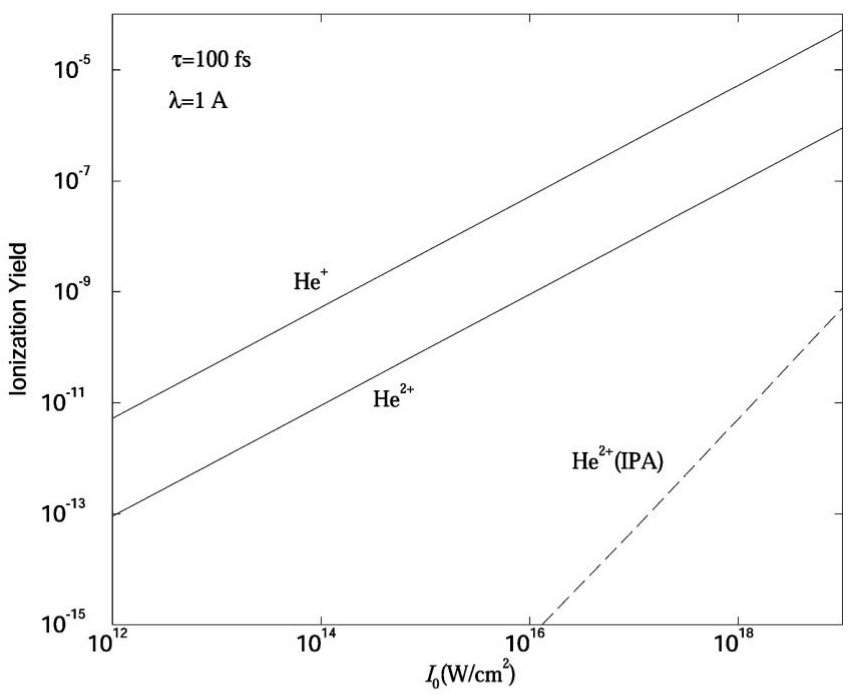

(a)



(b)

Figure 2

Ionization yield as a function of peak intensity for photon wavelength $1 \AA$ and pulse duration 100 fs. (a) Formation of $\mathrm{He}^{+}$and $\mathrm{He}^{2+} ;(b)$ formation of $\mathrm{Xe}^{+}$ and $\mathrm{Xe}^{2+}$. The dashed line denotes the use of an independent-particle approximation (IPA) for the transition for formation of a doubly charged state. 
These machines are expected to deliver photon intensities of about $10^{16} \mathrm{~W} \mathrm{~cm}^{-2}$ in $100 \mathrm{fs}$ at photon wavelengths down to $1 \AA$. There are a number of areas of atomic physics in which the availability of these sources may be of interest, and we have considered some of them here. Topics such as the creation of multiple-charged states, the competition between post-collisional effects and strong-field effects, and studies of hollow atoms under the exotic conditions of the XFEL parameters are some of the problems that we feel are the most promising. An overview of these topics was given in $\$ 2$.

In $\S 3$, some of these questions have been addressed for the rare gases, for which numerical estimates have been given. Although our discussion has concentrated on the particular case of the synchrotron sources at DESY and SLAC, which are expected to deliver the shortest wavelengths in the near future, many of the issues considered in this work are also applicable to other short-wavelength sources in the XUV that are under development in various laboratories.

This work was supported by the Division of Chemical Sciences, Office of Science, US Department of Energy.

\section{References}

Åberg, T. (1970). Phys. Rev. A, 2, 1726-1729.

Åberg, T. (1994). Nucl. Instrum. Methods B, 87, 5-16.

Azuma, Y., Hasegawa, S., Koike, F., Kutluk, G., Nagata, T., Shigemasa, E., Yagishita, A. \& Sellin, I. A. (1995). Phys. Rev. Lett. 74, 3768-3771.

Bambynek, W., Crasemann, B., Fink, R. W., Freund, H.-U., Mark, H., Swift, C. D., Price, R. E. \& Rao, P. V. (1972). Rev. Mod. Phys. 44, 716-813.

Becker, A. \& Faisal, M. (1999). Phys. Rev. A, 59, R1742-R1745.

Berger, M. J. \& Hubbell, J. H. (1987). XCOM: Photon Cross Section Database, Web Version 1.2, available at http://physics.nist.gov/xcom. (National Institute of Standards and Technology, Gaithersburg, MD 20899, August 1999.) Originally published as NBSIR 87-3597, Photon Cross Sections on a Personal Computer.

Blewett, J. P. (1946). Phys. Rev. 69, 87-95.

Brewczyk, M. \& Rzazewski, K. (1999). J. Phys. B, 32, L1-L4.

Briand, J. P., de Billy, L., Charles, P., Essabaa, S., Briand, P., Geller, R., Desclaux, J. P., Bliman, S. \& Ristori, C. (1990). Phys. Rev. Lett. 65, 159-162. Brinkmann, R., Materlik, G., Rossbach, J. \& Wagner, A. (1997). Editors. Conceptual Design of a $500 \mathrm{GeV} e^{+} e^{-}$Linear Collider with Integrated X-ray Laser Facility, DESY Report No. 1997-048, ch. 5. DESY, Hamburg, Germany.

Carlson, T. A. \& Nestor, C. W. Jr (1973). Phys. Rev. A, 8, 2887-2894.

Colson, W. B. (1997). Nucl. Instrum. Methods A, 393, 6-8.

Crance, M. (1987). Phys. Rep. 144, 117-185.

Crance, M. \& Aymar, M. (1985). J. Phys. (Paris), 46, 1887-1896.

DESY (2001). Free Electron Laser, http://www-hasylab.desy.de/facility/fel/ main.htm; TESLA, http://tesla.desy.de/new_pages/TDR_CD/start.html.

Diamant, R., Huotari, S., Kämälainen, K., Kao, C. C. \& Deutsch, M. (2000a). Phys. Rev. Lett. 84, 3278-3281.

Diamant, R., Huotari, S., Kämälainen, K., Kao, C. C. \& Deutsch, M. (2000b). Phys. Rev. A, 62, 052519.

Doniach, S. (2000). J. Synchrotron Rad. 7, 116-120.
Gajda, M., Krzywiński, J., Pluciński, L. \& Piraux, B. (2000). J. Phys. B, 33, 1271-1277.

Gavrila, M. (1992). Editor. Atoms in Intense Laser Fields. New York: Academic Press.

Geltman, S. (1985). Phys. Rev. Lett. 54, 1909-1912.

Geltman, S. \& Zakrzewski, J. (1988). J. Phys. B, 21, 47-62.

Journel, L., Cubaynes, D., Bizau, J.-M., Moussalami, A., Rouvellou, B., Wuilleumier, F., Voky, L., Faucher, P. \& Hibbert, A. (1996). Phys. Rev. Lett. 76, 30-33.

Kanter, E. P., Dunford, R. W., Krässig, B. \& Southworth, S. H. (1999). Phys. Rev. Lett. 83, 508-511.

Kapteyn, H. C. \& Falcone, R. W. (1988). Phys. Rev. A, 37, 2033-2038.

Kapteyn, H. C., Lee, R. W. \& Falcone, R. W. (1986). Phys. Rev. Lett. 57, 2939 2942.

Karule, E. (1990). Adv. At. Mol. Opt. Phys. 27, 265-299.

Keldysh, L. V. (1965). Sov. Phys. JETP, 20, 1307-1314.

Kiernan, L. M., Kennedy, E. T., Mosnier, J-P., Costello, J. T. \& Sonntag, B. F. (1994). Phys. Rev. Lett. 72, 2359-2362.

Kochur, A. G., Sukhorukov, V. L., Dudenko, A. I. \& Demekhin, Ph. V. (1995). J. Phys. B, 28, 387-402.

Lambropoulos, P., Maragakis, P. \& Zhang, J. (1998). Phys. Rep. 305, 203-293.

McGuire, J. H. (1997). Electron Correlation Dynamics in Atomic Collisions. Cambridge University Press.

McGuire, J. H., Itza-Ortiz, S., Godunov, A. L., Ederer, D. L., Wang, J. \& Burgdörfer, J. (2000). Phys. Rev. A, 62, 012702.

McPherson, A., Luk, T. S., Thompson, B. D., Borisov, A. B., Shiryaev, O. B., Chen, X., Boyer, K. \& Rhodes, C. K. (1994). Phys. Rev. Lett. 72, 1810-1813.

McPherson, A., Thompson, B. D., Borisov, A. B., Boyer, K. \& Rhodes, C. K. (1994). Nature (London), 370, 631-634.

Madsen, L. B., Schlagheck, P. \& Lambropoulos, P. (2000). Phys. Rev. Lett. 85, $42-45$.

Moribayashi, K., Sasaki, A. \& Tajima, T. (1998). Phys. Rev. A, 58, 2007-2015.

Moshammer, R., Schmitt, W., Ullrich, J., Kollmus, H., Cassimi, A., Dörner, R., Jagutzki, O., Mann, R., Olson, R. E., Prinz, H. T., Schmidt-Böcking, H. \& Spielberger, L. (1997). Phys. Rev. Lett. 79, 3621-3624.

Mukoyama, T. (1986). J. Phys. Soc. Jpn, 55, 3054-3058.

Novikov, S. A. \& Hopersky, A. N. (2000). J. Phys. B, 33, 2287-2294.

Novikov, S. A. \& Hopersky, A. N. (2002a). J. Phys. B. 35, L339-L343.

Novikov, S. A. \& Hopersky, A. N. (2002b). Radiat. Phys. Chem. 63, 115.

Parker, J. S., Moore, L. R., Meharg, K. J., Dundas, D. \& Taylor, K. T. (2001). J. Phys. B, 34, L69.

Perry, M. D., Landen, O. L., Szöke, A. \& Campbell, E. M. (1988). Phys. Rev. A, 37, 747-760.

SLAC (2001). Linac Coherent Light Source, http://www-ssrl.slac.stanford.edu/ $\mathrm{lcls} /$; Overview of projects, http://www.er.doe.gov/production/bes/BESAC/ PPT10-10-00.htm.

Tatchyn, R., Arthur, J., Baltay, M., Bane, K., Boyce, R., Cornacchia, M., Cremer, T., Fisher, A., Hahn, S.-J., Hernandez, M., Loew, G., Miller, R., Nelson, W. R., Nuhn, H.-D., Palmer, D., Paterson, J., Raubenheimer, T., Weaver, J., Wiedemann, H., Winick, H., Pellegrini, C., Travish, G., Scharlemann, E. T., Caspi, S., Fawley, W., Halbach, K., Kim, K.-J., Schlueter, R., Xie, M., Meyerhofer, D., Bonifacio, R. \& De Salvo, L. (1996). Nucl. Instrum. Methods A, 375, 274-283.

Tawara, H., Hayaishi, T., Koizumi, T., Matsuo, T., Shima, K., Tonuma, T. \& Yagishita, A. (1992). J. Phys. B, 25, 1467-1473.

Tschentscher, Th. \& Suortti, P. (1998). J. Synchrotron Rad. 5, 286-292.

Walker, B., Sheehy, B., DiMauro, L. F., Agostini, P., Schafer, K. J. \& Kulander, K. C. (1994). Phys. Rev. Lett. 73, 1227-1230.

Winick, H. (1998). J. Synchrotron Rad. 5, 168-175.

Winter, H. \& Aumayr, F. (1999). J. Phys. B, 32, R39-R65.

Zernik, W. (1964). Phys. Rev. A, 135, 51-57. 